\title{
Erratum zu: Mathe mit der Matte - Verkörperlichtes Training basisnumerischer Kompetenzen
}

\author{
Tanja Link - Eva Johanna Schwarz - Stefan Huber · Ursula Fischer • \\ Hans-Christoph Nuerk · Ulrike Cress · Korbinian Moeller
}

Online publiziert: 12 . Oktober 2015

(C) Springer Fachmedien Wiesbaden 2015

\section{Erratum zu: Z Erziehungswiss (2014) 17:257-277 DOI 10.1007/s11618-014-0533-2}

Leider haben wir es bei der Analyse der Daten versäumt, die in die Kovarianzanalysen (ANCOVA) eingehenden Kontrollvariablen zu zentrieren. Dadurch verändern sich im Hauptartikel die statistischen Kennwerte des Haupteffekts in der Analyse der differentiellen Trainingseffekte des allgemeinen, am Ausgangswert standardisierten

Die Onlineversion des Originalbeitrages ist erreichbar unter doi:10.1007/s11618-014-0533-2

Zusatzmaterial online Zusätzliche Information ist in der online Version dieses Artikels (doi:10.1007/s11618-015-0657-z) enthalten.

T. Link $(\bowtie) \cdot$ E. J. Schwarz · Prof. Dr. H.-C. Nuerk Psychologisches Institut, Eberhard Karls Universität, Schleichstraße 4, 72076 Tübingen, Deutschland

E-Mail: tanja.link@uni-tuebingen.de

E. J. Schwarz

E-Mail: eva-johanna.schwarz@student.uni-tuebingen.de

Prof. Dr. H.-C. Nuerk

E-Mail: hc.nuerk@uni-tuebingen.de

S. Huber · Dr. U. Fischer · Prof. Dr. U. Cress · Prof. Dr. K. Moeller

Leibniz-Institut für Wissensmedien,

Schleichstraße 6, 72076 Tübingen, Deutschland

E-Mail: s.huber@iwm-kmrc.de

Dr. U. Fischer

E-Mail: u.fischer@iwm-kmrc.de

Prof. Dr. U. Cress

E-Mail: u.cress@iwm-kmrc.de

Prof. Dr. K. Moeller

E-Mail: k.moeller@iwm-kmrc.de 
Schätzfehlers auf S. 269. Die Reanalyse der ANCOVA mit verbaler Arbeitsgedächtniskapazität ergab wie die ursprünglich berichteten Ergebnisse keinen signifikanten Haupteffekt $[F(1,41)=1,90, p=, 18]$.

$\mathrm{Zu}$ beachten ist, dass die Zentrierung der Kontrollvariablen zu Änderungen im Supplementary Material führten, in welchem die Wirkungen der beiden Kontrolltrainingsbedingungen getrennt voneinander mit der Wirkung des Experimentaltrainings verglichen wurden. Eine aktualisierte Version der Analysen und eine darauf abgestimmte Interpretation sind unter (10.1007/s11618-015-0657-z) zu finden.

Die Veränderungen betreffen zum einen den Haupteffekt in der Analyse der differentiellen Trainingseffekte in Bezug auf die Verbesserung der allgemeinen räumlichen Zahlengröße (nicht getrennt für inversions- und nicht-inversionsrelevante Items) im Vergleich der Experimentalbedingung mit der medienbezogenen Kontrollbedingung. Die zuvor gefundenen marginal signifikanten differentiellen Effekte, sowohl für die Veränderungen des absoluten Schätzfehlers als auch für die des am Ausgangswert standardisierten Schätzfehlers, konnten nicht mehr beobachtet werden. Die ANCOVA für den Vergleich der Veränderungen des absoluten Schätzfehlers, in welcher visuelle Arbeitsgedächtniskapazität als Kontrollvariable berücksichtigt wurde, ergab keinen signifikanten Haupteffekt der Trainingsbedingung mehr $[F(1,18)=1,34, p=, 26]$. Ebenso ergab die ANCOVA, die zum Vergleich der Veränderungen des am Ausgangswert standardisierten Schätzfehlers berechnet wurde, keinen signifikanten Haupteffekt der Trainingsbedingung mehr $[F(1,16)=0,79, p=, 39]$. Hierbei wurden generelle kognitive Fähigkeiten, arithmetische Leistungen und verbale Arbeitsgedächtniskapazität als Kontrollvariablen berücksichtigt.

In der spezifischen Analyse der Trainingseffekte getrennt für inversionsrelevante und nicht-inversionsrelevante Zielzahlen veränderte die Zentrierung der Kontrollvariablen die Ergebnisse nur unwesentlich. Für die Analyse der differentiellen Trainingseffekte in der Gruppe mit aufgabenbezogenem Kontrolltraining wurde in Bezug auf die Verbesserung des absoluten Schätzfehlers inversionsrelevanter Zielzahlen eine ANCOVA mit der Kontrollvariablen generelle kognitive Fähigkeiten berechnet. Diese ergab wie ursprünglich berichtet einen marginal signifikanten Haupteffekt der Trainingsbedingung $[F(1,20)=3,30, p=, 08]$, der darauf hindeutete, dass die Kinder ihre Schätzfehler bei inversionsrelevanten Zielzahlen durch das Experimentaltraining tendenziell stärker verbessern konnten $(M=3,10)$ als durch das Kontrolltraining $(M=0,15)$.

Im Vergleich der Experimentalbedingung mit der medienbezogenem Kontrollbedingung wurden differentielle Veränderungen der absoluten Schätzfehler nicht-inversionsrelevanter Zielzahlen in einer ANCOVA mit visueller Arbeitsgedächtniskapazität als Kovariate überprüft. Diese ergab auch nach der Zentrierung keinen signifikanten Haupteffekt für die Trainingsbedingung $[F(1,19)=0,47, p=, 50]$. In Bezug auf die Verbesserung der am Ausgangswert standardisierten Schätzfehler nicht-inversionsrelevanter Zielzahlen wurde ebenfalls eine ANCOVA durchgeführt, in welcher die Kontrollvariablen generelle kognitive Fähigkeiten und verbale Arbeitsgedächtniskapazität berücksichtigt wurden. Auch nach der Zentrierung der Kontrollvariablen ließ sich kein signifikanter Haupteffekt der Trainingsbedingung beobachten $[F(1,18)=1,33, p=, 26]$. 